\title{
Clinical Characteristics and Outcomes of First 100 Severe Acute Respiratory Syndrome Coronavirus-2 (SARS-Cov-2) Patients: A Single Center Experience
}

\author{
Kashif Naeem, Vinod Choondal, Mahmoud Hamouri, Ahmed Abbas, Sreevidya Machingal, Farzeela Fahad, \\ Kusay Almusa, Mona Osman, Afaf Ashoor, Mahdi Sial, Mohammad Ali, Eslam Siddiq, Waleed Flayyih, and \\ Najeeba Abdulrazzaq
}

\begin{abstract}
Since December 2019, the world has witnessed the Coronavirus disease caused by the Severe Acute Respiratory Syndrome Coronavirus-2, which has been declared a pandemic by the World Health Organization in March 2020. We share our initial experience at a center in Dubai, UAE, with a diverse ethnic population and present the clinical characteristics of the first 100 laboratory-confirmed coronavirus disease patients. In this retrospective, single center study, we included all adult $(\geq \mathbf{1 2}$ years old) laboratory-confirmed COVID-19 patients who presented at Al Kuwait Hospital, Dubai, UAE between 21 February 2020 and 15 April 2020. We extracted data on the demograhics, clinical presentation, laboratory and imaging results, treatment, complications and outcomes from the electronic medical records. Results are expressed as counts and percentages for categorical variables, and mean (with range) for continuous variables. A total of one hundred patients were studied. Mean age was 44 years (range $13-82$ years); $16 \%$ were aged more than 60 years. $69 \%$ were males. Most of the patients (41\%) belonged to South Asia, while $33 \%$ belonged to the Middle East. $21 \%$ were diabetics, $20 \%$ were hypertensives, $10 \%$ were active smokers and $6 \%$ were known cases of asthma/chronic obstructive lung disease. Upon admission, fever $(46 \%)$ and dry cough $(41 \%)$ were the most common symptoms, while $24 \%$ were asymptomatic on admission. Mean duration of symptoms before hospital admission was 5.1 days (range 1-14 days). Upon admission, $8 \%$ had low platelets, $7 \%$ had lymphopenia, $61 \%$ had high $\mathrm{C}$-reactive protein, $48 \%$ had high ferritin, $37 \%$ had high lactate dehydrogenase, and $31 \%$ had high D-dimers. $63 \%$ had normal chest radiography upon presentation. Computed tomography chest showed ground glass opacification in $80 \%$, consolidation in $21 \%$ while $14 \%$ had ill defined patchy opacities. All lesions were located peripherally and $79 \%$ had bilateral involvement with predominantly lower lobe disease. $8 \%$ had critical illness. Chloroquine/hydroxychloroquine $(\mathbf{9 3} \%)$ and the protease inhibitor lopinavir-ritonavir $(86 \%)$ were the most commonly prescribed treatment. $8 \%$ needed non-invasive ventilation and $7 \%$ were intubated and ventilated invasively. $7 \%$ developed acute repiratory distress syndrome, $5 \%$ went into septic shock and needed vasopressor support, $2 \%$ developed acute cardiac injury, $17 \%$ had acute kidney injury, $11 \%$ had acute liver injury and $2 \%$ developed disseminated intravascular coagulation. Almost half of the patients $(49 \%)$ were declared recovered after having two negative COVID-19 PCR tests while 5\% died. We concluded that the Coronavirus disease 2019 (COVID-19) presents with different clinical characteristics in the UAE with an ethnic diverse background. Majority affected were young, males and diabetic. One-fourth were asymptomatic on
\end{abstract}

admission, while fever and dry cough were the most common symptoms. High C-reactive protein and ferritin on admission was common. Most of the patients had normal chest radiograph on admission, while computed tomography chest showed the characteristic findings in over two-thirds. Almost half of our patients recovered while $5 \%$ died. This is an intial experience only and increased patient cohort will provide further information.

Index Terms - Clinical characteristics; Coronavirus disease 2019; Severe acute respiratory syndrome coronavirus- 2 .

\section{INTRODUCTION}

Human coronaviruses are pathogens that have been largely known to cause the 'common cold' in otherwise healthy people. However, the epidemiology changed significantly when, in the 21 st century, two highly pathogenic coronaviruses - Severe Acute Respiratory Syndrome (SARS) coronavirus and Middle East Respiratory Syndrome (MERS) coronavirus - emerged to cause global epidemics with alarming morbidity and mortality [1,2]. In December 2019, a new strain of coronavirus, officially named Severe Acute Respiratory Syndrome Coronavirus 2 (SARS-Cov-2), was first isolated from three patients with Coronavirus disease 2019 (COVID-19) by the Chinese Center for Disease Control and Prevention $[3,4]$, connected to the cluster of acute respiratory illness cases from Wuhan, China. On 30 January 2020, the World Health Organization declared that the outbreak of COVID-19 constituted a Public Health Emergency of International Concern [5]. Subsequently, based on the high levels of global spread and the severity of COVID-19, on 11 March 2020, the Director-General of the World Heart Organisation declared the COVID-19 outbreak a pandemic [6]. As of 11 May 2020, the total number of confirmed cases worldwide are 4,013,728, with 278,993 confirmed deaths among the 215 countries affected (World Health Organisation). 
United Arab Emirates (UAE) has a unique population that comprises people of various ethnic backgrounds and is home to over 200 nationalities. We have 18,198 confirmed cases with 198 reported deaths according to the World Health Organisation data (as of 11 May 2020). Our center, Al Kuwait Hospital, Dubai began to receive confirmed cases from 21 February 2020. Owing to the diverse population and paucity of local data, we aim to share our initial experience while managing this disease and present data on the clincial characteristics of 100 patients including the demographics, symptomatology, laboratory/radiological findings, treatment and outcomes.

\section{METHODS}

The study was conducted at Al Kuwait Hospital, Dubai, UAE between 21 February 2020 and 15 April 2020. Ethical approval was obtained from Dubai Research and Ethics Committtee. Informed patient consent was waived as data collection was largely retrospective.

\section{A. Study participants:}

This study was a retrospective analysis of all confirmed SARS-Cov-2 patients who were admitted to our center between 21 February 2020 and 15 April 2020. We included all consecutive patients who were age 12 years or more and had a laboratory confirmed positive Polymerase Chain Reaction (PCR) result irrespective of the ethnic background. We excluded patients who were suspected of COVID-19 infection but had not undergone PCR testing. The laboratory used Sacace Real Time Reverse Transcription Polymerase Chain Reaction (rRT-PCR) test to diagnose COVID-19 disease. The test was performed on patients' nasopharyngeal swabs. RNA was extracted using SaMag Viral Nucleic Acid Extraction system. Extracted RNA was amplified using BGIReal Time Fluorescent RT-PCR kit for the detection of COVID-19. After the initial diagnosis, the tests were repeated during the hospital stay every 72 hours until two consecutive negative samples were achieved. Transfers from other centers within the same health authority were considered a single visit. Descriptive statistics were used to summarize the data. Results are expressed as counts and percentages for categorical variables, and mean (with range) for continuous variables.

\section{B. Data sources:}

We extracted the data from the electronic medical records of the hospital that stores the daily record keeping and all investigations. We obtained information about the patient demographics, history, laboratory tests, radiological investigations, treatment, complications and outcomes. Details of patient symptoms and duration, exposure history, ethnicity and comorbidities were also noted. Laboratory tests recorded on admission included full blood count, coagulation profile, creatinine and electrolytes, liver enzymes, inflammatory markers (C-reactive protein, procalcitonin, ferritin, d-dimers, lactate dehydrogenase) and cardiac markers (high-sensitivity troponin, pro-B type natriuretic peptide). We reviewed admission chest radiography and high-resolution chest computed tomography (HRCT) with the radiologist to record accurate findings. Detailed records of in-patient treatment were obtained for each patient. Complications relating to multi-organ failure and clinical outcomes were also noted.

\section{Study outcomes:}

All patients were followed for deterioration in clinical outcomes. This included the need for ventilation (invasive and non-invasive), development of acute respiratory distress syndrome, septic shock with need for vasopressors, acidosis, acute cardiac/kidney/liver injury and disseminated intravascular coagulation. Discharge outcomes were recorded as deaths, recovered cases and transfer to another isolation facilty.

\section{Study definitions:}

Septic shock was defined as a clinical construct of sepsis with persisting hypotension requiring vasopressors to maintain MAP $\geq 65 \mathrm{~mm} \mathrm{Hg}$ and having a serum lactate level $>2 \mathrm{mmol} / \mathrm{L} \quad(18 \mathrm{mg} / \mathrm{dL})$ despite adequate volume resuscitation [7]. Acute respiratory distress syndrome was defined as per the Berlin Definition; the development of bilateral lung opacities (not fully explained by effusions, lobar/lung collapse, or nodules), respiratory failure not fully explained by cardiac failure or fluid overload and low $\mathrm{PaO} 2 / \mathrm{FiO} 2$, within 1 week of a known clinical insult or new or worsening respiratory symptoms [8]. Acute kidney injury was defined as any of the following: increase in serum creatinine $\geq 0.3 \mathrm{mg} / \mathrm{dL}(\geq 26.5 \mathrm{umol} / \mathrm{L})$ within $48 \mathrm{hrs}$; or increase in serum creatinine $\geq 1.5$ times baseline, which is known or presumed to have occurred within the prior 7 dasys; or urine volume $<0.5 \mathrm{ml} / \mathrm{kg} / \mathrm{hr}$ for 6 hours [9]. Acute liver injury was defined as the presence of high alanine amiotransferase (ALT) and/or high aspartate aminotransferase (AST) more than 5 times the upper normal limit. Acute cardiac injury was defined as the presence of high-sensitivity troponin-I level above the 99th percentile upper reference limit. Acidosis was defined as $\mathrm{pH}<7.35$ on an arterial blood gas sample. Type 2 respiratory failure was defined as an arterial oxygen tension $(\mathrm{Pa}, \mathrm{O} 2)$ of $<8.0 \mathrm{kPa}(60$ $\mathrm{mmHg})$, an arterial carbon dioxide tension $(\mathrm{Pa}, \mathrm{CO} 2)$ of $>6.0$ $\mathrm{kPa} \quad(45 \mathrm{mmHg}) \quad[\mathbf{1 0} . \quad$ Disseminated intravascular coagulation was defined in the context of sepsis as an acquired syndrome characterized by activation of coagulation pathways, resulting in the formation of intravascular thrombi and depletion of platelets and coagulation factors, leading to decreased platelet count, increased prothrombin time and elevated d-dimers. Recovered (from infection) was defined by two consecutive negative PCR tests with an interval between the two tests of at least 48 hours, in the absence of COVID-19 related symptoms [11]. Disease severity was classified as mild (non-pneumonia or mild pneumonia), severe (dyspnea, respirartory rate $\geq 30 / \mathrm{min}$, blood oxygen 
saturation $\leq 93 \%$, ratio of partial pressure of arterial oxygen $(\mathrm{PaO} 2)$ to fraction of inspired oxygen $(\mathrm{FiO} 2)<300$, lung infiltrates $>50 \%$ within $24-48$ hours), and critical (respiratory failure, septic shock, multiorgan dysfunction/failure) [12].

\section{RESULTS}

One hundred patients were studied. All had laboratory confirmed COVID-19 disease.

\section{A. Clinical data:}

Table 1 describes the clinical data for 100 patients. Mean age was 44 years (range 13-82 years). 16\% were aged more than 60 years. $69 \%$ were males. Regarding ethnic backgrounds, most of the patients (41\%) belonged to South Asia (India, Pakistan, Bangladesh and Nepal), while 33\% belonged to the Middle East. $28 \%$ had positive travel history while $38 \%$ tested positive after they were screened due to the history of close contacts with confirmed COVID-19 patients. Regarding the comorbidities, $21 \%$ were diabetics, $20 \%$ were hypertensives and $10 \%$ were active smokers. $6 \%$ were known cases of asthma/chronic obstructive disease. Upon admission, fever $(46 \%)$ and dry cough (41\%) were the most common symptoms reported. $24 \%$ were asymptomatic on admission. Mean duration of symptoms before hospital admission was 5.1 days (range 1-14 days). Measurement of vital signs upon admission showed that $5 \%$ had temperature $>38 \mathrm{C}, 5 \%$ presented with low systolic blood pressure (SBP) $<110$ $\mathrm{mmHg}, 24 \%$ had high SBP >140 mmHg, 9\% had tachypnea with respiratory rate 20 or more, $4 \%$ had tachycardia, and only $1 \%$ had low oxygen saturations $<93 \%$.

Table 1: Clinical characteristics of 100 SARS-CoV-2 positive patients.

\begin{tabular}{|l|l|}
\hline Characteristic & No. of patients (total=100) \\
\hline Age: mean (range), years & $44(13-82)$ \\
\hline - 60 years, no. (\%) & $16(16)$ \\
\hline Males: no. (\%) & $69(69)$ \\
\hline Regions according to nationality: no. (\%) & \\
Middle East & $33(33)$ \\
South Asia & $41(41)$ \\
South-East Asia & $5(5)$ \\
East Asia & $2(2)$ \\
West Europe & $2(2)$ \\
Africa & $10(10)$ \\
\hline Positive travel history: no. (\%) & $3(3)$ \\
\hline Positive contact history: no. (\%) & $28(28)$ \\
\hline Comorbidities: no. (\%) & $38(38)$ \\
Diabetes Mellitus & \\
Hypertension & \\
Smoker & $21(21)$ \\
Chronic kidney disease & $20(20)$ \\
Ischemic heart disease & $10(10)$ \\
Asthma/COPD & $2(2)$ \\
Hypothyroidism & $1(1)$ \\
Pregnancy & $6(6)$ \\
\hline Symptom onset to hospital admission: days, mean (range) & $5(5)$ \\
\hline Symptoms on admission: no. (\%) & $3(3)$ \\
None & $5.1(1-14)$ \\
Fever & \\
Dry cough & $24(24)$ \\
Shortness of breath & $46(46)$ \\
Sore throat & $41(41)$ \\
Runny nose & $14(14)$ \\
\hline & $18(18)$ \\
& $12(12)$ \\
\hline
\end{tabular}

\begin{tabular}{|l|l|}
\hline Rigors/chills & $7(7)$ \\
Bodyaches & $12(12)$ \\
Headache & $14(14)$ \\
Nausea/vomiting & $3(3)$ \\
Diarrhoea & $3(3)$ \\
Loss of smell/taste & $1(1)$ \\
\hline & \\
Vital signs on admission: no. (\%) & $5(5)$ \\
Temperature $>38 \mathrm{C}$ & $5(5)$ \\
Systolic blood pressure $\quad>110 \mathrm{mmHg}$ & $24(24)$ \\
& $9(9)$ \\
Respiratory rate $\geq 20 /$ minute $\quad>140 \mathrm{mmHg}$ & $4(4)$ \\
Pulse $>100 /$ minute & $1(1)$ \\
Oxygen saturations $<93 \%$ & \\
& \\
&
\end{tabular}

\section{B. Laboratory and imaging data:}

Table 2 describes the laboratory and imaging results of 100 patients upon admission. $6 \%$ were anemic with hemoglobin les sthan $11 \mathrm{~g} / \mathrm{dL}$. 8\% had leucopenia while $5 \%$ had leucocytosis. $8 \%$ had low platelets while $7 \%$ had lymphopenia. Regarding the inflammatory markers, Creactive protein was high in $61 \%$, while procalcitonin was high in $10 \%$. 48\% had high ferritin, 37\% had high lactate dehydrogenase., and $31 \%$ had high D-dimers. $6 \%$ had estimated GFR $<60 \mathrm{ml} / \mathrm{min} / 1.73 \mathrm{~m}^{2} .34 \%$ had high alanine aminotrasferase. Cardiac markers showed high troponin-I in $4 \%$ and high pro-BNP in $16 \%$.

All patients had chest radiography on admission and high resolution computed tomography (HRCT) chest within 48 hours. $63 \%$ had normal chest radiography upon admission, $37 \%$ had abnormal findings, and $11 \%$ developed abnormalities after an initial normal $\mathrm{x}$ ray during the hospital stay. In patients who presented with an abnormal xray, 64\% showed fine reticular opacities while $39 \%$ had patchy opacities. The lesions predominantly affected lower zones (92\%) and peripheral lung fields (100\%). HRCT chest was abnormal in $76 \%$ upon admission. The most common finding was ground glass opacification seen in $80 \%$ of abnormal CT scans. Consolidation was present in $21 \%$ while $14 \%$ had ill defined patchy opacities. All lesions were located peripherally and $79 \%$ had bilateral involvement with predominantly lower lobe disease.

Table 2: Laboratory and imaging findings of 100 SARS-CoV-2 positive patients.

\begin{tabular}{|l|l|}
\hline Characteristic & $\begin{array}{l}\text { No. of patients: } \\
\text { no./total no. (\%) }\end{array}$ \\
\hline $\begin{array}{l}\text { Time taken to recover: } \\
\text { Time taken from 1st positive PCR result to 2nd consecutive } \\
\text { negative PCR result, days, mean (range) }\end{array}$ & $12.3(5-26)$ \\
\hline
\end{tabular}




\begin{tabular}{|c|c|c|}
\hline \multicolumn{3}{|c|}{ Laboratory parameters: } \\
\hline \multirow{3}{*}{$\begin{array}{l}\text { Hemoglobin } \\
\text { White cell count }\end{array}$} & $<11 \mathrm{~g} / \mathrm{dL}$ & $6 / 100(6)$ \\
\hline & $<4 \times 10^{3} / \mathrm{mcL}$ & $8 / 100(8)$ \\
\hline & $>11 \times 10^{3} / \mathrm{mcL}$ & $5 / 100(5)$ \\
\hline \multicolumn{2}{|c|}{ 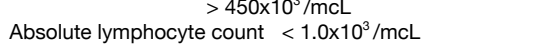 } & $\begin{array}{l}1 / 100(1) \\
7 / 100(7)\end{array}$ \\
\hline \multicolumn{2}{|c|}{ International normalised ratio $>1.3$} & $1 / 76(1)$ \\
\hline \multicolumn{2}{|c|}{ C-reactive protein $\leq 3 \mathrm{mg} / \mathrm{L}$} & $39 / 100(39)$ \\
\hline & $3 \mathrm{mg} / \mathrm{L}$ & $61 / 100(61)$ \\
\hline \multicolumn{2}{|c|}{ Procalcitonin $>0.10 \mathrm{ug} / \mathrm{L}$} & $8 / 78(10)$ \\
\hline \multicolumn{2}{|c|}{ Sodium $\quad<135 \mathrm{mmol} / \mathrm{L}$} & $\begin{array}{l}11 / 100(11) \\
1 / 100(1)\end{array}$ \\
\hline \multicolumn{2}{|c|}{$\begin{array}{ll}\text { Potassium } & <3.5 \mathrm{mmol} / \mathrm{L} \\
& >5 \mathrm{mmol} / \mathrm{L}\end{array}$} & $\begin{array}{l}13 / 100(13) \\
2 / 100(2)\end{array}$ \\
\hline \multicolumn{2}{|c|}{ Urea $>8 \mathrm{mmol} / \mathrm{L}$} & $7 / 100(7)$ \\
\hline \multicolumn{2}{|c|}{ Creatinine $>110 \mathrm{umol} / \mathrm{l}$} & $9 / 99(9)$ \\
\hline \multicolumn{2}{|c|}{$\begin{array}{l}\text { Estimated glomerular filtration rate }<60 \mathrm{ml} / \mathrm{min} / 1.73 \mathrm{~m}^{2} \\
\text { Bilirubin }>10 \mathrm{umol} / \mathrm{a}\end{array}$} & $6 / 99(6)$ \\
\hline \multicolumn{2}{|c|}{ Bilirubin $>10 u m o l / 1$} & $17 / 100(17)$ \\
\hline \multicolumn{2}{|c|}{ Alanine amiotransferase $>50 \mathrm{IU} / \mathrm{L}$} & $34 / 100(34)$ \\
\hline \multicolumn{2}{|c|}{ Aspartate aminotransferase $>50 \mathrm{IU} / \mathrm{L}$} & $14 / 100(14)$ \\
\hline \multicolumn{2}{|c|}{ Alkaline phosphatase $>150 \mathrm{IU} / \mathrm{L}$} & $5 / 100(5)$ \\
\hline \multicolumn{2}{|c|}{ Albumin $<30 \mathrm{gm} / \mathrm{L}$} & $8 / 100(8)$ \\
\hline \multirow{2}{*}{\multicolumn{2}{|c|}{$\begin{array}{l}\text { D-dimer }>0.5 \mathrm{mg} / \mathrm{dL} \\
\text { Ferritin }>250 \mathrm{mcg} / \mathrm{L}\end{array}$}} & 15/48 (31) \\
\hline & & 25/52 (48) \\
\hline \multicolumn{2}{|c|}{ High-sensitivity troponin $\quad>60 \mathrm{ng} / \mathrm{L}$} & $2 / 49(4)$ \\
\hline \multicolumn{2}{|c|}{ Total creatine kinase $\quad>200 \mathrm{IU} / \mathrm{L}$} & $4 / 42(10)$ \\
\hline \multicolumn{2}{|c|}{ Pro-B type natriuretic peptide $>200 \mathrm{ng} / \mathrm{L}$} & $5 / 32(16)$ \\
\hline \multicolumn{2}{|c|}{ Lactate dehydrogenase $>225 \mathrm{IU} / \mathrm{L}$} & $27 / 70(37)$ \\
\hline Chest $x$ ray findi & gs on admission: & \\
\hline Normal on admis & & 60/96 (63) \\
\hline Abnormal on adn & sion & $36 / 96(37)$ \\
\hline Abnormal after in & al normal xray & $11 / 96(11)$ \\
\hline Findings: & & \\
\hline Fine reticular infil & ates & $23 / 36(64)$ \\
\hline Patchy opacities & & $14 / 36(39)$ \\
\hline Bilateral & & $17 / 36(47)$ \\
\hline Predominantly lo & er zones & 33/36 (92) \\
\hline Peripheral & & $36 / 36(100)$ \\
\hline Central/hilar & & $3 / 36(8)$ \\
\hline Pleural effusion & & 2/36 (5) \\
\hline $\begin{array}{l}\text { High resolution } \\
\text { (within } 48 \mathrm{hrs} \text { ) : }\end{array}$ & omputed tomography (HRCT) chest & \\
\hline Normal & & 23/94 (24) \\
\hline Abnormal & & $71 / 94(76)$ \\
\hline Findings: & & \\
\hline Ground glass ope & ification & $57 / 71(80)$ \\
\hline Consolidation & & $15 / 71(21)$ \\
\hline ill-defined patchy & pacities & 10/71 (14) \\
\hline Septal thickening & & 4/71 (6) \\
\hline Bilateral & & $56 / 71$ (79) \\
\hline Peripheral & & $71 / 71$ (100) \\
\hline Predominantly lo & er lobes & 33/71 (46) \\
\hline Pleural thickening & & $2 / 71$ (3) \\
\hline Pleural effusion & & 4/71 (6) \\
\hline Lymphadenopath & & 3/71 (4) \\
\hline
\end{tabular}

C. Severity, treatment, complications and outcomes data:

Table 3 describes the details of disease severity, in-patient treatment given, complications and clinical outcomes of 100 patients. Most of the patients (79\%) had mild disease, $13 \%$ had severe disease and $8 \%$ had critical illness. The medications were prescribed according to the local guidelines. The most commonly prescibed treatment was the antimalarial drug chloroquine/hydroxychloroquine (93\%) and the protease inhibitor combination lopinavir-ritonavir $(86 \%)$, followed by intravenous antibiotics $(21 \%)$, interferonalpha $(16 \%)$, oral azithromycin $(12 \%)$, ribavirin $(9 \%)$, intravenous steroids $(9 \%)$, favipiravir $(7 \%)$, interleukin-6 receptor antagonist tocilizumab (7\%), and the antifungal drug anidulafungin (4\%). 20\% needed face mask oxygen to maintain oxygen saturations, while $8 \%$ needed non-invasive ventilation and $7 \%$ were intubated and ventilated invasively. 1 patient needed continous renal replacement therapy. 13\% were admitted to the intensive care unit (ICU) once they fulfilled the criteria for ICU admission as per local guidelines. $7 \%$ developed acute repiratory distress syndrome, 5\% went into septic shock and needed vasopressor support. $2 \%$ developed acute cardiac injury, $17 \%$ had acute kidney injury, $11 \%$ had acute liver injury and $2 \%$ developed disseminated intravascular coagulation. Almost half of the patients (49\%) were declared recovered after having two negative COVID19 PCR tests while 5\% died.

Table 3: Severity, Treatment, complications and outcomes of 100 SARSCoV-2 positive patients.

\begin{tabular}{|l|l|}
\hline Characteristic & $\begin{array}{l}\text { No. of } \\
\text { (total=100) } \\
\mathbf{n}(\%)\end{array}$ \\
\hline Severity of illness: & \\
Mild & $79(79)$ \\
Severe & $13(13)$ \\
Critical & $8(8)$ \\
\hline In-patient treatment: & \\
Chloroquine/hydroxychloroquine & $93(93)$ \\
Lopinavir+Ritonavir (Kaletra) & $86(86)$ \\
Favipiravir & $7(7)$ \\
Interferon-alpha & $16(16)$ \\
Ribavirin & $9(9)$ \\
Oseltamivir & $4(4)$ \\
Oral azithromycin & $12(12)$ \\
Intravenous antibiotics & $21(21)$ \\
Intravenous steroids & $9(9)$ \\
Anidulafungin & $4(4)$ \\
Tocilizumab & $7(7)$ \\
Face mask oxygen & $20(20)$ \\
Non-invasive ventilation & $8(8)$ \\
Invasive ventilation & $7(7)$ \\
Continous renal replacement therapy & $1(1)$ \\
(CRRT) & \\
\hline Complications during hospital stay: & \\
\hline Admission to intensive care unit (ICU) & $13(13)$ \\
Septic shock & $5(5)$ \\
Intravenous vasopressors & $5(5)$ \\
Acute respiratory distress syndrome & $7(7)$ \\
Type 2 respiratory failure & $7(7)$ \\
Acute cardiac injury & $2(2)$ \\
Acidosis & $6(6)$ \\
Acute kidney injury & $17(17)$ \\
Acute liver injury & $11(11)$ \\
Disseminated intravascular coagulation & $2(2)$ \\
Gastro-intestinal bleeding & $1(1)$ \\
\hline Outcomes at data cutoff: & \\
Recovered & $49(49)$ \\
Death & $5(5)$ \\
Transferred to another isolation facility & $32(32)$ \\
Still admitted & $14(14)$ \\
& \\
\hline
\end{tabular}

\section{DISCUSSION}

This retrospective study is the first from the middle east region that provides an insight to an intial experience of a single center in managing the first 100 laboratory confirmed Covid-19 patients.

Mean age of patients in our study was 44 years with a range from 13-82 years. This indicates a predominantly young population as only $16 \%$ were aged more than 60 years. Earliest data from China where they performed a descriptive, exploratory analysis of all cases diagnosed as of February 11 , 2020 , found that among the 44,672 confirmed cases, most were aged 30-79 years [13]. However, among the 5,700 COVID-19 patients studied in the New York area, median age was 63 years, interquartile range 52-75 and range 0-107 years [14]. 69\% were males in our study, hence the conclusions drawn may not be applicable to women. UAE hosts an ethnically diverse population that is reflected in the patient cohort. Most patients (41\%) belonged to South-Asia while $33 \%$ belonged to the Middle East. Our study does not 
analyse data according to the ethnic backgound as findings are presented taking the whole cohort together. We found out that among our patients, $21 \%$ were diabetic, $20 \%$ hypertensives and $10 \%$ smokers. Only $6 \%$ had previous history of asthma/COPD. In a systemic review and meta analysis of seven studies [15], the most prevalent comorbidities were hypertension $(21.1 \%$, 95\% CI: 13.0 $27.2 \%)$ and diabetes $(9.7 \%, 95 \%$ CI: $7.2-12.2 \%)$, followed by cardiovascular disease $(8.4 \%, 95 \% \mathrm{CI}: 3.8-13.8 \%)$ and respiratory system disease $(1.5 \%, 95 \% \mathrm{CI}: 0.9-2.1 \%)$. When compared between severe and non-severe patients, the pooled odds ratio of hypertension, respiratory system disease, and cardiovascular disease were 2.36 (95\% CI: 1.46-3.83), 2.46 (95\% CI: 1.76-3.44) and 3.42 (95\% CI: 1.88-6.22) respectively. The study from New York area reported hypertension (56.6\%), obesity (41.7\%) and diabetes $(33.8 \%)$ as the most noted comorbidities [14].

We looked at the time from symptom onset to hospital admission that was found to be a mean of 5.1 days. Still, 24\% of our patients were asymptomatic on admission, hence, providing some indication of the incubation period of the virus. Regarding the symptoms on admission, we found that most patients presented with fever $(46 \%)$ and dry cough (41\%). Guan et al [16] analysed 1,099 COVID-19 patients and found fever (44\%), cough (68\%) and dyspnea (19\%) as common symptoms. Yangab et al [15] reported in a meta analysis of 1,576 infected patients, fever $(91.3 \%, 95 \% \mathrm{CI}$ : 86-97\%), cough $(67.7 \%$, 95\% CI: 59-76\%), fatigue $(51.0 \%$, 95\% CI: $34-68 \%)$ and dyspnea (30.4\%, 95\% CI: $21-40 \%)$ as the most common symptoms.

A significant number of hematological and biochemical abnormalities were detected in our patients on admission laboratory testing. $7 \%$ had lymphopenia, $8 \%$ had thrombocytopenia and $6 \%$ had anemia. Of these, lymphopenia and thrombocytopenia have been associated with worse prognosis [17]. Guan et al [16] reported lymphopenia in $83 \%$, thrombocytopenia in $36 \%$, and leukopenia in 34\%. Huang et al [18] and Wang et al [19] have reported an association between lymphopenia and the need for intensive care, while $\mathrm{Wu}$ et al [20] reported an association between lymphopenia and the developement of acute respiratory distress syndrome. A meta analysis of nine studies has suggested that thrombocytopenia is significantly associated with the severity of COVID-19 disease [21]. Regarding the inflammatory markers, our study also showed that on admission $61 \%$ had high C-reactive protein (CRP), $10 \%$ had high pro-calcitonin and $37 \%$ had high lactate dehydrogenase (LDH). The high CRP might even indicate a co-existent bacterial infection in these patients. These findings correlate remarkably well with the findings from Guan et al [16] who reported high CRP in $60.7 \%$, high procalcitonin in $5.5 \%$, high LDH in $41 \%$ of patients and high ferritin in 48\%. Wu et al [20] have reported high CRP, ferritin and LDH to be associated with the development of acute respiratory distress syndrome, intensive care and even mortality. Coagulation abnormalities are frequently found in severe COVID-19 patients. Our study showed high d-dimers in $31 \%$. In a multicenter retrospective cohort study, increases d-dimer levels $(>1 \mathrm{ug} / \mathrm{ml})$ were significantly associated with in-hospital death in the multivariate analysis $(\mathrm{p}=0.003)$ [22]. We performed chest radiography on admission for all and high-resolution CT scan of chest within 48 hours. Comparing the two modalities, CT chest has proven to be more specific and sensitive to the extent that CT findings may be present even before symptoms, and may be diagnostic in falsenegative PCR tested patients [23]. Our study showed that the majority $(63 \%)$ presented with a normal chest radiograph, while $11 \%$ developed abnormal findings after initial normal radiograph. Therefore, we concluded that chest radiography is more appropriate to follow the progression of disease, while CT chest provided characteristic diagnostic information. We found that the most common finding on chest CT scan was ground glass opacification (80\%), followed by consolidation (21\%). All lesions involved lung periphery, $79 \%$ bilateral, with $46 \%$ affecting the lower lobes predominantly. A systematic review of 919 COVID-19 patients [24] showed that the characteristic findings included ground glass opacification $(88.0 \%)$, bilateral involvement $(87.5 \%)$, peripheral distribution $(76.0 \%)$, and multilobar involvement $(78.8 \%)$. We found that $79 \%$ of our patients belonged to the mild severity of illness; $13 \%$ severe; and $8 \%$ critical.

The patients were treated as per the local guidelines. We used oral chloroquine phosphate or hydroxychloroquine or lopinavir-ritonavir for 5 days for any symptomatic laboratory-confirmed COVID-19 patient without pneumonia. In the presence of pneumonia, the drugs are combined and given for 7 days; additionally favipiravir and remdesivir is considered. In critically ill patients, in addition to above, tocilizumab and interferon-alfa is considered. Most of our patients received chloroquine/hydroxychloroquine (93\%) and lopinavir-ritonavir (86\%). Additionally, $7 \%$ had favipiravir, $16 \%$ had interferon-alfa, $12 \%$ oral azithromycin, $9 \%$ intravenous steroids, 7\% tocilizumab. We understand that limited trial data exists for the suggested current treatment. A double-blind randomised phase 2 trial in Brazil [25] evaluated high versus low dosages of chloroquine where the high dose regimen was discontinued due to cardiotoxicity. In a randomised, parallel group study in 31 patients with COVID-19 pneumonia, hydroxychloroquine was associated with early symptom relief [26]. A randomised, open-label trial of 199 severe COVID-19 patients showed that lopinavirritonavir did not reduce time to clinical improvement or other events [27].

Our study showed that $8 \%$ patients had critical illness based on pre-defined criteria. Overall, $8 \%$ needed bilevel positive airway pressure (BiPAP) while $7 \%$ were intubated and ventilated to maintain ventilation. As for the complications, 5\% had septic shock needing vasopressors, $7 \%$ developed acute respiratory distress syndrome and type 2 respiratory failure, $17 \%$ had acute kidney injury, $11 \%$ had acute liver injury, $2 \%$ had acute cardiac injury. In a study of 
5,700 patients [14], 14\% were treated in intensive care, $12 \%$ needed invasive mechanical ventilation and $21 \%$ died. In a small study of 21 critically ill patients with COVID-19 [28], $19 \%$ needed non-invasive positive pressure ventilation, $71 \%$ needed mechanical ventilation, vasopressors were used for $67 \%$, 57\% developed acute respiratory distress syndrome, $19 \%$ had acute kidney injury, 33\% had cardiomyopathy and $14 \%$ developed acute hepatic injury; 52\% died. Our study also found out that almost half of the patients (49\%) recovered as proved by laboratory confirmed negative PCR tests, while $5 \%$ died.

The study has limitations. This is a retrospective analysis of a small cohort from a single center. However, this is the first initial experience reported from the Gulf region and highlights the clinical characteristics of the disease in this region.

\section{CONCLUSION}

COVID-19 presents with different clinical characteristics in the UAE with an ethnic diverse background. Majority affected were young, males and diabetic. One-fourth were asymptomatic on admission, while fever and dry cough were the most common symptoms. High CRP and ferritin on admission is common. Most of the patients had normal chest radiograph on admission, while CT chest showed the characteristic findings in over two-thirds. Almost half of our patients recovered while $5 \%$ died. This is an initial experience only and increased patient cohort will provide further information.

\section{FUNDING}

The study is not funded.

\section{CONFLICT OF INTEREST}

All authors have no conflict of interest to declare.

\section{REFERENCES}

[1] Drosten C, Günther S, Preiser W, etal . Identification of a nove coronavirus in patients with severe acute respiratory syndrome. $\mathrm{N}$ Engl J Med 2003;348:1967-76.

[2] Zaki AM, van Boheemen S, Bestebroer TM, Osterhaus AD, Fouchier RA. Isolation of a novel coronavirus from a man with pneumonia in Saudi Arabia. N Engl J Med 2012;367:1814-20.

[3] Zhu N, Zhang D, Wang W, etal. China Novel Coronavirus Investigating and Research Team. A Novel Coronavirus from Patients with Pneumonia in China, 2019. N Engl J Med 2020;382:727-33.

[4] Gralinski LE, Menachery VD. Return of the Coronavirus: 2019nCoV. Viruses 2020;12:E135.

[5] World Health Organization. Statement on the second meeting of the International Health Regulations (2005) Emergency Committee regarding the outbreak of novel coronavirus (2019-nCoV). Geneva: WHO; 2020.

[6] World Health Organization. WHO Director-General's opening remarks at the media briefing on COVID-19 - 11 March 2020: WHO 2020

[7] The Third International Consensus Definitions for Sepsis and Septic Shock, JAMA, 2016;315(8):801-810.

[8] Acute Respiratory Distress Syndrome: The Berlin Definition, JAMA 2012;307(23):2526-2533
[9] Kidney Disease Improving Global Outcomes (KDIGO) Clinical Practice Guideline for Acute Kidney Injury. Kidney Int Suppl. 2012;2:1-138.

[10] Roussos. C, Koutsoukou, A. Respiratory failure. European Respiratory Journal 2003 22: 3s-14s.

[11] European Society of Cardiology guidance for the diagnosis and management of CV disease during the COVID-19 pandemic.

[12] Novel Coronavirus Pneumonia Emergency Response Epidemiology Team. Vital surveillances: the epidemiological characteristics of an outbreak of 2019 novel coronavirus disease - China, 2020. China CDC Weekly.

[13] The Novel Coronavirus Pneumonia Emergency Response Epidemiology Team. The Epidemiological Characteristics of an Outbreak of 2019 Novel Coronavirus Diseases (COVID-19) - China, 2020[J]. China CDC Weekly, 2020, 2(8): 113-122. doi: $10.46234 / \mathrm{ccdcw} 2020.032$.

[14] Richardson S, Hirsch JS, Narasimhan M, Crawford JM, McGinn T, Davidson KW et al. Presenting Characteristics, Comorbidities, and Outcomes Among 5700 Patients Hospitalized with COVID-19 in the New York City Area. JAMA. 2020 Apr 22. doi: 10.1001/jama.2020.6775.

[15] Yang J, Zheng Y, Gou X, Wang H, Wang Y, Zhou Y, Pu K et al. Prevalence of comorbidities and and its effects in patients infected with SARS-CoV-2: a systematic review and meta-analysis. International Journal of Infectious Diseases 2020;94:91-95.

[16] Guan WJ, Ni ZY, Hu Y. Clinical characteristics of coronavirus disease 2019 in China. N Engl J Med 2020;382:1708-1720.

[17] Terpos E, Ntanasis-Stathopoulos I, Elalamy I, Kastritis E, Segentanis $\mathrm{T}$, Politou $\mathrm{M}$ et al. Hematological findings and complications of COVID-19. American Journal of Hematology 2020.

[18] Huang C, Wang Y, Li X, et al. Clinical features of patients infected with 2019 novel coronavirus in Wuhan, China. Lancet 2020 Feb 15; 395(10223): 497-506.

[19] Wang $\mathrm{D}, \mathrm{Hu} \mathrm{B}, \mathrm{Hu} \mathrm{C}$, et al. Clinical Characteristics of 138 Hospitalized Patients With 2019 Novel Coronavirus-Infected Pneumonia in Wuhan, China. JAMA 2020 Feb 7.

[20] Wu C, Chen X, Cai Y, et al. Risk Factors Associated With Acute Respiratory Distress Syndrome and Death in Patients With Coronavirus Disease 2019 Pneumonia in Wuhan, China. JAMA Intern Med 2020 Mar 13.

[21] Lippi G, Plebani M, Henry BM. Thrombocytopenia is associated with severe coronavirus disease 2019 (COVID-19) infections: A metaanalysis. Clin Chim Acta 2020 Mar 13; 506: 145-148.

[22] Zhou F, Yu T, Du R, et al. Clinical course and risk factors for mortality of adult inpatients with COVID-19 in Wuhan, China: a retrospective cohort study. Lancet 2020 Mar 28; 395(10229): 1054-1062

[23] Huang P, Liu T, Huang L, et al. Use of chest CT in combination with negative RT-PCR assay for the 2019 novel coronavirus but high clinical suspicion. Radiology 2020 Feb 12.

[24] Salehi S, Abedi A, Balakrishnan S, Gholamrezanezhad A. Coronavirus disease 2019: a systematic review of imaging findings in 919 patients. American Journal of Radiology 2020;215:1-7.

[25] Borba MGS, Val FFA, Sampaio VS. Effect of high versus low dosages of chloroquine diphosphate as adjunctive therapy for patients hospitalized with severe acute respiratory syndrome coronavirus- 2 infection: a randomised clinical trial. JAMA 2020;3:e208857.

[26] Chen Z, Hu J, Zhang Z, Jiang S, Han S, Yan D. Efficacy of hydroxychloroquine in patients with COVID-19: results of a randomised clinical trial. MedRxiv 2020.03.22.20040758.

[27] Cao B, Wang Y, Wen D. A trial of Lopinavir-Ritonavir in adults hospitalized with severe Covid-19. N Engl J Med. 2020.

[28] Arentz M, Yim E, Klaff L, Lokhandwala S, Riedo F, Chong M et al. Characteristics and outcomes of 21 critically ill patients with Covid19 in Washington state. JAMA 2020. 


\section{FIRST AUTHOR BIOGRAPHY}

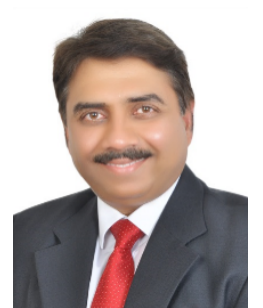

Dr Kashif Bin Naeem [MBBS, MRCP (UK), CESR

Cardiology (UK)] is currently working as a Consultant Cardiologist at Al Kuwait Hospital, Dubai, UAE. After completing MBBS from Pakistan, he moved to UK where he completed his Residency in Internal Medicine from London and achieved MRCP (UK) in 2007. He pursued his career in Non-invasive Cardiology and was awarded CESR, CCT equivalent, in Cardiology by the General Medical Council/Royal College of Physicians, UK in 2018. His work affiliation includes Guy's and St. Thomas' Hospitals, London; London Chest Hospital; Royal Free Hospital, London; and King Fahad Medical City, Riyadh. He specializes in Advanced Echocardiography and been awarded Diplomate American Board of Echocardiography. He is a member of British Cardiovascular Society, European Society of Cardiology (ESC), Emirates Cardiac Society, Acute Cardiovascular Care Association (ESC) and European Association of Cardio Vascular Imaging. $\mathrm{He}$ is an active researcher; published several papers in peer-reviewed journals and presented abstracts in European Cardiology conferences. He has keen interest in voluntary work where he offers free consultation at medical camps, World Heart Day and World Hypertension Day events; and visits schools to educate students. He is affiliated with the University of Sharjah, UAE to teach year 5 medical students. His current interests include preventive cardiology and echocardiography and has delivered talks at different platforms. 